\title{
Cornelis A. 'Neil' Gehrels
}

\author{
Neil Gehrels passed away on 6 February 2017. A pioneer of observational high-energy astrophysics, he \\ was an exceptional leader, scholar, colleague and friend.
}

T he scientific community suffered a tremendous blow with the death of Neil Gehrels, who passed away on 6 February 2017 after a battle with pancreatic cancer. Through his research and leadership, he was a driving force in gamma-ray astrophysics up to his death but also worked in the diverse fields of planetary sciences, galaxy evolution and gravitational wave astronomy.

Niel grew up in a scientific family. His father, Tom Gehrels, himself an eminent scientist, was instrumental in propelling the University of Arizona into a powerhouse in astronomy and planetary sciences. In spite of this, Neil did not initially imagine a career in science, his early passion being music. As an undergraduate music student at the University of Arizona he aimed to hone his skills playing clarinet, guitar and piano - his goal was to become a classical music composer. Later he added physics as a double major, and became enamoured of research. Neil took this newfound love to Caltech, where he pursued his $\mathrm{PhD}$ in Caltech's Space Radiation Laboratory with the noted physicists Rochus 'Robbie' Vogt and Ed Stone. Neil's first project was to calibrate a cosmic-ray instrument on Voyager. In 1979, when Voyager flew past Jupiter, Neil analysed the data from this instrument, making the surprise discovery of oxygen and sulfur in the planet's magnetosphere. Gehrels and Stone deduced that the particles were expelled by volcanos on Jupiter's moon Io, a discovery that was a highlight of the Voyager mission, and remains one of Neil's most-cited results.

It was at Caltech where Neil met his life partner, Ellen Williams. Ellen and Neil (both given Caltech's distinguished alumni award last year) were married on campus in 1980, and moved to Maryland two years later. Ellen joined the University of Maryland as a professor of physics, and Neil went to the Goddard Space Flight Center, where he spent the rest of his career.

At Goddard Neil turned his attention to gamma-ray astrophysics, a field which, with crude instruments and just a handful of known cosmic sources, was in its infancy. Neil led a team that developed a balloon-borne gamma-ray spectrometer that made detailed observations of the

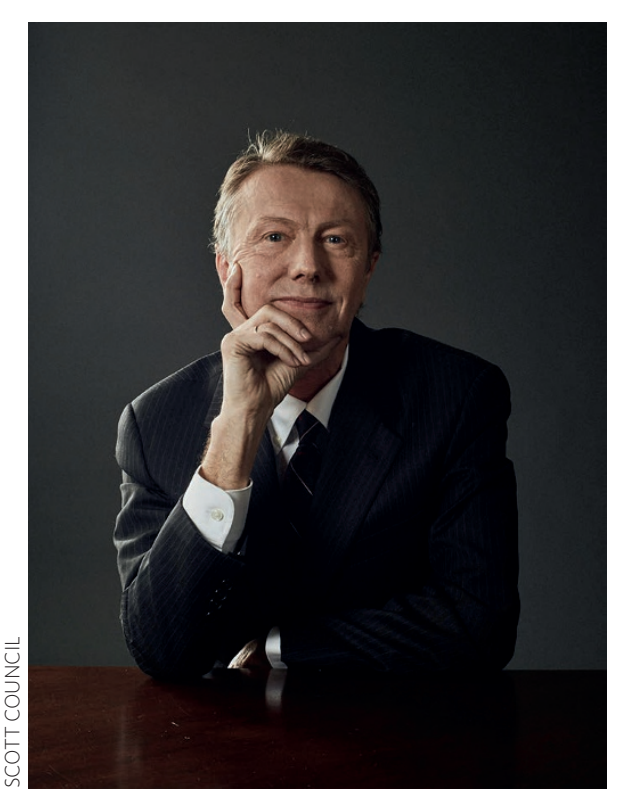

Cornelis A. 'Neil' Gehrels (1952-2017)

centre of the Milky Way at energies in the range $100 \mathrm{keV}$ to $10 \mathrm{MeV}$. This experiment detected broadened radioactive decay lines of ${ }^{56} \mathrm{Co}$ from the famous SN1987A supernova, providing the first evidence that the material ejected by the demise of this massive star was strongly mixed and asymmetric - clues central to understanding the explosion mechanism.

Neil then moved on to leadership positions on a series of gamma-ray space missions. He was the project scientist of the Compton Gamma Ray Observatory (CGRO), the first mission in the line of four NASA Great Observatories that established that gamma-ray bursts - bright flashes of energetic radiation lasting a few seconds result from two distinct populations. By the end of CGRO's mission in 2000, the census of cosmic gamma-ray sources had gone from a few to many thousands. Neil described this time of discovery as one of the best in his professional life. Neil went on to be US Mission Scientist of the ESA's INTEGRAL mission, and Deputy Project Scientist for NASA's Fermi Gamma-ray Space Telescope.

Neil's crowning achievement in highenergy astrophysics was his role as Principal
Investigator of the Swift Gamma-Ray Burst Mission. Neil led Swift from its inception, through development and, more than twelve years after its launch in 2004, to Swift's position as one of NASA's most scientifically productive missions. Swift was initially dedicated to gamma-ray burst studies, and it made many important discoveries, including the first identification of afterglows of short-duration events. The mission broadened its observational scope over time, becoming a powerful tool for studies of a wide range of transient phenomena, from supernovae to neutron stars and black holes. Neil oversaw this transformation, always enthusiastic and excited about new scientific directions. For his role on Swift he was awarded (with the Swift team) the Bruno Rossi Prize of the American Astronomical Society, and the Henry Draper Medal from the National Academy of Sciences. Very shortly before his death he was awarded the prestigious Dan David Prize for his role in the up-andcoming field of transient astrophysics.

In the last decade of his career Neil began to devote his talents outside of high-energy astrophysics. As a result of early involvement with the Joint Dark Energy Mission he became the project scientist for the Wide Field Infrared Survey Telescope, NASA's next flagship to follow the James Webb Space Telescope. He was also a core member of the LIGO Scientific Collaboration. For his contributions to science, he was inducted into the American Academy of Arts and Sciences and the National Academy of Sciences.

Although his time was cut short, Neil lived a full life. He was an incredibly productive scientist, with over 750 publications. Outside the office, Neil was an avid mountaineer - almost every trip to a scientific conference was combined with the ascent of some high peak. In 2006 and again in 2015 Neil climbed the Nose route on El Capitan in Yosemite in six-day solo ascents. Neil devoted significant time to scientific advisory roles, where his thoughtful, deliberative style was highly valued. Together with his family - Ellen and their two children, Thomas and Emily - he volunteered in disadvantaged Maryland communities, and in 2005 Neil 
helped establish an internship program for impoverished local high school students to work in his labs.

Neil was disarmingly friendly, unassuming, and one of the most genuine people I have ever met. He led large project teams harmoniously and with grace, managing difficult situations calmly and wisely, but with the firmness necessary to lead a project forward. He had an infectious enthusiasm for all science, and in particular he would go out of his way to find out what young researchers were up to - frequenting the poster sessions at conferences and getting to know the students who were using the satellites he had helped to lead. Those who met him quickly counted him as a colleague, then soon after a friend.

Neil, private about his illness, worked until shortly before his death, and many in the community are still reeling from the news of his passing. But we celebrate this life lived fully, and Neil's legacy will live on with each new discovery from the missions he helped to create.

FIONA HARRISON is at the California Institute of Technology, 290-17, Pasadena, California 91125, USA.

e-mail:fiona@srl.caltech.edu 